\title{
A case of prolonged delirium tremens
}

\author{
Jerome $\underline{\text { Nicholas }}^{1}$, MBBs, Rajesh $\underline{\text { Jacob }}^{1,2,}$ MBBS, MD, Rochelle $\underline{K i n s o n}^{3}$, MB BCh BAO, MMed
}

ABSTRACT We present a case of delirium tremens lasting for five weeks in an alcohol-dependent individual. The patient required high-dose benzodiazepines, which is atypical and rare. The clinical presentation and management of this patient is discussed.

Keywords: alcohol withdrawal, benzodiazepines, prolonged delirium tremens

\section{INTRODUCTION}

Delirium tremens, the most serious manifestation of alcohol withdrawal, occurs in approximately $5 \%$ of hospitalised patients with alcohol dependence and has a reported mortality rate approaching $15 \% .{ }^{(1)}$ The current diagnostic criteria for delirium tremens include disturbance of consciousness, change in cognition or perceptual disturbance developing in a short period, and emergence of symptoms during or shortly after withdrawal from heavy alcohol intake. Delirium tremens usually lasts for approximately 5-7 days, and prolonged delirium tremens is rare, usually occurring only if there are underlying medical causes. There are only a few cases of prolonged delirium tremens treated with high-dose benzodiazepines described in the literature.(2) Herein, we report the case of a patient with prolonged delirium tremens that lasted for 35 days, requiring high-dose benzodiazepines. The patient's delirium tremens resolved with no major underlying medical complicating factors.

\section{CASE REPORT}

A 35-year-old Chinese man presented to the outpatient addiction clinic at the Institute of Mental Health (IMH) requesting for help for his alcohol addiction. On assessment, the patient gave a history of alcohol consumption since the age of 18 years; initially, he only drank socially, but gradually progressed to develop a dependence pattern characterised by craving, intolerance, withdrawal symptoms, salience, as well as blackouts and episodes of withdrawal seizures for about five years.

The patient was last seen at the outpatient addiction clinic on July 1, 2011, during which he was observed to have tremors. His last drink was 24 hours prior to that visit. He was prescribed intramuscular lorazepam $4 \mathrm{mg}$ stat and three days of tapering doses of lorazepam, and was asked to attend an outpatient appointment after three days. Although inpatient admission is recommended for detoxification, as outpatient detoxification is unsafe when there are high risk factors such as a past history of withdrawal seizures, it was not done in this case.
On July 2, the patient presented to the emergency room with coarse tremors, complaints of visual hallucinations (he saw snakes coming out of the window), an unsteady gait and disorientation. In view of the possibility of delirium tremens, he was transferred to the general hospital where he was then admitted to the medical ward on the same day and administered intravenous thiamine and fluids, as well as oral lorazepam. These medications were later switched to intravenous diazepam $10 \mathrm{mg}$ every six hours. His liver function tests revealed elevated bilirubin and mildly elevated aspartate transaminase. Alkaline phosphatase and alanine transaminases were normal, and there was no evidence of hepatic encephalopathy or liver flap. As the patient continued to show signs of delirium tremens with low blood pressure, he was transferred to the medical intensive care unit on July 5 . He was then weaned off intravenous diazepam and put on oral diazepam $20 \mathrm{mg}$ every four hours (i.e. $120 \mathrm{mg}$ daily). The patient's creatine kinase was found to be elevated, and the possibility of rhabdomyolysis was kept in mind since the patient was being restrained for agitated behaviour. His creatine kinase level gradually declined, and he was transferred back to the general medical ward on July 12 .

The patient was reviewed on a regular basis by the liaison psychiatrist, who documented persistent signs of delirium tremens in the form of disorientation, fluctuating consciousness, and visual and auditory hallucinations. The patient was started on oral haloperidol $0.5 \mathrm{mg}$ twice daily, and his alcohol withdrawal scores on a rating scale were continuously monitored. Due to the patient's agitated behaviour, computed tomography was not carried out; however, previous magnetic resonance imaging done in March 2010 was unremarkable. On July 18, the patient's dose of diazepam was decreased to $17.5 \mathrm{mg}$ every four hours. Abdominal ultrasonography revealed hepatic cirrhosis with portal hypertension and no ascites. Endoscopy revealed oesophageal varices and gastritis.

The patient was transferred back to IMH on July 22 for further management. He continued to show signs of delirium tremens, but was less agitated. Mental status examination ${ }^{1}$ Department of General Psychiatry, Institute of Mental Health, ${ }^{2}$ NUS-YLL School of Medicine, Duke-NUS Graduate Medical School, ${ }^{3}$ Department of Psychological Medicine,
Tan Tock Seng Hospital, Singapore
Correspondence: Adj Asst Prof Rajesh Jacob, Consultant Psychiatrist, Institute of Mental Health, 10 Buangkok View, Singapore 539747. Jacob_rajesh@imh.com.sg 
in the emergency room at $\mathrm{IMH}$ on the same day revealed irrelevant speech, auditory hallucinations and disorientation to time and place. He was admitted to the general psychiatric ward and assessed by the ward team on a regular basis. On July 26 , he was referred to the addictions team that regularly reviewed him. It was noted that the patient continued to exhibit signs of delirium tremens in the form of disorientation and perceptual disturbances.

The patient's delirium tremens finally resolved on August 5, 35 days after its onset. Mental status examination on the same day revealed intact orientation to time, place and person, with no perceptual disturbances. He was transferred to the addictions unit for further management and rehabilitation, where he expressed an interest to try medications to suppress his cravings for alcohol and was motivated to give up alcohol completely in view of his underlying liver cirrhosis.

\section{DISCUSSION}

This case illustrates a prolonged course of delirium tremens, which lasted for 35 days. Prolonged delirium tremens is quite rare and not many cases have been reported in the literature. Delirium tremens usually lasts for 5-7 days, with onset between 48-72 hours after the last drink in patients with alcohol dependence. ${ }^{(3)}$ The duration of delirium tremens can be prolonged if there are underlying medical causes such as infections, electrolyte abnormalities or pneumonia. ${ }^{(4)}$ In our patient, rhabdomyolysis, which was diagnosed and resolved quickly, may have contributed to the delirium. However, the patient's delirium continued to persist even after his creatine kinase level normalised. He was also diagnosed with liver cirrhosis and portal hypertension, but there was no evidence of hepatic encephalopathy or any variceal bleeds that may have resulted in the prolonged course of delirium tremens. No other possible medical causes of delirium tremens were identified.

Another feature of our case was that relatively high doses of diazepam were administered to our patient - starting with intravenous diazepam, followed by oral diazepam $120 \mathrm{mg}$ daily in divided doses. Diazepam was only tapered off on Day 34 of delirium tremens. There was no clinical evidence of Wernicke's encephalopathy or Korsakoff's psychosis. Other than diazepam, the use of propofol to manage refractory delirium tremens has also been reported.(5)

Our case highlights that delirium tremens can last longer than the usual 5-7 day duration and adds to the limited existing literature on this rare phenomenon. It also underlines that high doses of benzodiazepines can be used over a protracted period of time to manage this condition successfully. However, it is important to exclude underlying medical causes that can prolong delirium tremens.

\section{REFERENCES}

1. Mayo-Smith MF, Beecher LH, Fischer TL, et al. Management of alcohol withdrawal delirium. An evidence-based practice guideline. Arch Intern Med 2004; 164:1405-12.

2. Wolf KM, Shaughnessy AF, Middleton DB. Prolonged delirium tremens requiring massive doses of medication. J Am Board Fam Pract 1993; 6:502-4.

3. Guthrie SK. The treatment of alcohol withdrawal. Pharmacotherapy 1989; 9:131-43.

4. Miller FT. Protracted alcohol withdrawal delirium. J Subst Abuse Treat 1994; 11:127-130.

5. Mahajan R, Singh R, Bansal PD, Bala R. Use of Propofol as adjuvant therapy in refractory delirium tremens. Ind Psychiatry J 2010; 19:58-9. 